\title{
Characterization and optical properties of erbium oxide doped ZnO-SLS glass for potential optical and optoelectronic materials
}

\begin{abstract}
Erbium doped $\mathrm{ZnO}-\mathrm{SLS}$ (soda lime silica) glass system have been prepared by the conventional melt quenching technique. The physical, structural and optical properties are explained by analysing the data obtained from Energy Dispersive X-ray Fluorescence (EDXRF), density $(\rho)$, molar volume ( $\mathrm{V} \mathrm{m}$ ), X-ray diffraction (XRD), fourier transform infra-red (FTIR) and UV-visible (UV-Vis) results. The measured physical parameters like density and molar volume are found to vary linearly and exponentially with increasing Er2O3 content, respectively. X-ray powder diffractrogram show broad peaks which conforms glassy nature of the sample. FTIR spectroscopy reveals the presence of $\mathrm{SiO} 4, \mathrm{ZnO} 4$ and $\mathrm{Er}-\mathrm{O}$ vibration groups in the glass samples. The optical absorption spectra were measured in the wavelength range from 300 to $800 \mathrm{~nm}$ and the optical band gaps were determined. The optical absorption spectra of Er3+ ions in these glasses show three bands and are assigned to the transitions level. It was found that the optical band gap decreases from 3.083 to $3.037 \mathrm{eV}$ with an increase in Er2O3 concentration.
\end{abstract}

Keyword: Glasses; Soda lime silica; Erbium oxide; Optical materials; Optical properties 